\title{
Probing the Mechanisms of an Air Amplifier Using a LTQ-FT-ICR-MS and Fluorescence Spectroscopy
}

\author{
R. Brent Dixon, ${ }^{\text {a David C. Muddiman, }}{ }^{\text {a Adam M. Hawkridge, }}{ }^{\text {a }}$ \\ and A. G. Fedorov ${ }^{\text {b }}$ \\ ${ }^{a}$ W. M. Keck FT-ICR Mass Spectrometry Laboratory, Department of Chemistry, North Carolina State \\ University, Raleigh, North Carolina, USA \\ ${ }^{b}$ G. W. Woodruff School of Mechanical Engineering and Parker H. Petit Institute for Bioengineering \\ and Bioscience, Georgia Institute of Technology, Atlanta, Georgia, USA
}

We report the first quantitative assessment of electrosprayed droplet/ion focusing enabled by the use of a voltage-assisted air amplifier between an electrospray ionization emitter and a hybrid linear ion trap Fourier transform ion cyclotron resonance mass spectrometer (ESI-LTQFT-ICR-MS). A solution of fluorescent dye was electrosprayed with a stainless steel mesh screen placed in front of the MS inlet capillary acting as a gas-permeable imaging plate for fluorescence spectroscopy. Without use of the air amplifier, no detectable FT-ICR signal was observed, as well as no detectable fluorescence on the screen upon imaging using a fluorescence scanner. When the air amplifier was turned $\mathrm{ON}$ while electrospraying the fluorescent dye, FT-ICR mass spectra with high signal to noise ratio were obtained with an average ion injection time of $21 \mathrm{~ms}$ for an AGC target value of $5 \times 10^{5}$. Imaging of the screen using a fluorescence scanner produced a distinct spot of cross-sectional area $\sim 33.5 \mathrm{~mm}^{2}$ in front of the MS inlet capillary. These experimental results provide direct evidence of aerodynamic focusing of electrosprayed droplets/ions enabled by an air amplifier, resulting in improved electrospray droplet/ion capture efficiency and reduced ion injection time. A second set of experiments was carried out to explore whether the air amplifier assists in desolvation. By electrospraying a mix of quaternary amines, ratios of increasingly hydrophobic molecules were obtained. Observation of the solvophobic effect associated with electrospray ionization resulted in a higher abundance of the hydrophobic molecule. This bias was eliminated when the air amplifier was turned $\mathrm{ON}$ and a response indicative of the respective component concentrations of the molecules in the bulk solution was observed. (J Am Soc Mass Spectrom 2007, 18, 1909-1913) ( 2007 American Society for Mass Spectrometry

$\mathrm{I}$ $\mathrm{t}$ is well known that electrospray ionization is a soft ionization technique that enables the study of biological samples by mass spectrometry [1]. However, it is estimated that $99.9 \%$ or more of the electrosprayed ions are lost in transport to the detector [2-6]. A recent publication indicates that significant sampling efficiency is obtained with a short emitter to capillary distance, at the expense of lower ionization efficiency [7]. At longer distances, less sample is captured, but there is improved ionization due to more efficient sample desolvation. Based on the results presented herein, along with previous reports, the air amplifier has the capabilities to improve capture efficiency and sample desolvation. Devices such as the ion funnel [8-10], high-field asymmetric ion mobility spectrometry (FAIMS) [11-13], the air amplifier [14-16], and an interface plate [17] have been reported to improve ion

Address reprint requests to Dr. D. C. Muddiman, W. M. Keck FT-ICR Mass Spectrometry Laboratory, Department of Chemistry, North Carolina State University, Raleigh, NC 27695, USA. E-mail: david_muddiman@ncsu.edu abundance. To translate current technology into future advances in mass spectrometry, systematic investigations of devices such as the air amplifier are necessary to improve instrument performance and subsequently the study of biological systems. The purpose of this study is to explore whether focusing and desolvation are mechanisms of the air amplifier.

Previous reports have suggested that the use of an air amplifier results in higher analyte desolvation efficiency as evidenced by an increase in average chargestate [18]. Specifically for FT-ICR MS, this increase in average-charge state directly results in higher resolving power [19]. Additionally, these reports suggest that focusing of the ions is a possible mechanism for the increased ion abundance but, to date, detailed studies of these mechanisms are lacking. The aim of this work is to investigate droplet/ion focusing and analyte desolvation by the air amplifier as possible mechanisms for improved ionization efficiency and ion transmission. This is demonstrated by complimentary means of fluorescence spectroscopy imaging and mass spectrometric 
detection, giving explicit evidence of the air amplifier's capability to focus the electrosprayed ions towards the inlet of the mass spectrometer. The desolvation mechanism is probed using a set of molecules characteristic of their chemical properties of increasing hydrophobicity with increasing alkyl chain length.

\section{Experimental}

\section{Materials}

Rhodamine 6G (99\% purity), tetramethylammonium bromide $(98 \%$ purity), tetrabutylammonium bromide ( $98 \%$ purity), tetraheptylammonium bromide $(98 \%$ purity), and Fluka brand formic acid were obtained from Sigma-Aldrich (St. Louis, MO). HPLC grade acetonitrile and high purity water were purchased from Burdick and Jackson (Morristown, NJ). All reagents were used as received without further purification. Rhodamine $6 \mathrm{G}$ was brought to a final concentration of $2 \mu \mathrm{M}$ by serial dilution of a stock solution of $1 \mathrm{mg} / \mathrm{mL}$ in solvent composed of water and acetonitrile $(1: 1 \mathrm{vol} / \mathrm{vol})$ with $0.1 \%$ formic acid. A mix of quaternary amines was brought to a final concentration of $5 \mu \mathrm{M}$ in a solvent composed of water and acetonitrile $(19: 1 \mathrm{vol} / \mathrm{vol})$ with $0.1 \%$ formic acid by combining equal volumes of $5 \mu \mathrm{M}$ each of the quaternary amines. Tetramethylammonium bromide and tetrabutylammonium bromide were brought up in water and $0.1 \%$ FA. Tetraheptylammonium bromide was brought up in a solvent composed of water and acetonitrile $(17: 1 \mathrm{vol} / \mathrm{vol})$ with $0.1 \%$ formic acid (a small-volume of organic phase solvent was necessary to solvate the tetraheptylammonium due to its hydrophobicity).

\section{Experimental Apparatus}

Figure 1 schematically shows the electrospray ionization source, an air amplifier, and a gas-permeable

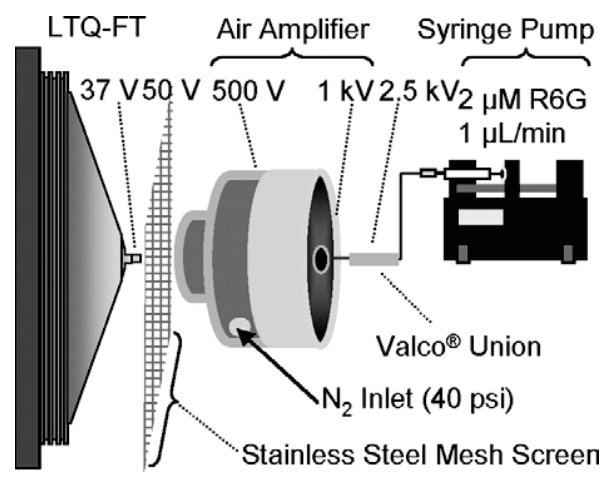

Figure 1. Schematic of the experimental set-up and operating conditions. A Thermo LTQ-FT interface is shown at the left with standard capillary. The gas-permeable stainless steel mesh screen is inserted between the MS capillary inlet and the voltage assisted air amplifier. A pressure of 40 psi (at the regulator) was used to induce flow in the air amplifier, resulting in aerodynamic focusing of the electrosprayed ions. The electrospray emitter and syringe pump supplying the sample are also shown at the right. stainless steel mesh screen in an aerial view of the experimental configuration together with experimental conditions; 304-stainless steel cloth with a mesh count of $60 \times 60$ per linear inch, an average wire diameter of $0.0075 \mathrm{in}$. and an opening width of $0.0090 \mathrm{in}$. was obtained from MSC Industrial Supply Co. (Atlanta, GA) and cut into portions for the experiments. The electrospray solution was infused at a rate of $1 \mu \mathrm{L} / \mathrm{min}$ through a $75 \mu \mathrm{m}$ i.d. fused silica capillary and $30 \mu \mathrm{m}$ tapered PicoTip (New Objective, Woburn, MA) using a Harvard PHD-2000 syringe pump. An air amplifier fabricated and implemented in the same fashion as the device previously described by Hawkridge et al. [15] was incorporated on the front end of a hybrid LTQ-FTICR mass spectrometer (Thermo Fisher, San Jose, CA) equipped with an Oxford Instruments actively shielded 7T superconducting magnet (Concord, MA). One major difference from the previous implementation of the device, specific only to the focusing experiments, was that a standard capillary was used to permit the screen to fit between the air amplifier and the MS inlet. This results in a long distance $(\sim 40 \mathrm{~mm})$ from ESI emitter to MS inlet. All mass spectra were collected with the mass spectrometer in the FT mode.

Throughout this paper, the air amplifier is said to be "ON" when metered $99.98 \%$ purity nitrogen at 40 psi (pressure at regulator) was continuously supplied to the device. The voltages applied to the air amplifier stages were held constant during the entire experiment at the values shown in Figure 1. The AGC target for the ICR cell was set at $5 \times 10^{5}$ and the maximum ion injection time was set to $1000 \mathrm{~ms}$. To simultaneously compare the fluorescent images for both the air amplifier OFF and the air amplifier $\mathrm{ON}$, a stainless steel mesh screen with dimensions of 2 in. $\times 3$ in. was implemented with 1.5 in. $\times 2$ in. dedicated to each phase of the experiment so that a single fluorescence scan would capture both phases of the experiment. A standard Thermo MS capillary interface was used to introduce ions into the mass spectrometer thus allowing the screen to fit between the outlet of the air amplifier and the MS capillary. It is important to note that the screen was biased at $50 \mathrm{~V}$ throughout the entire set of experiments to maintain a decreasing voltage gradient from the ESI emitter towards the MS inlet.

A BioRad Pharos FX Plus molecular imager (Hercules, $\mathrm{Ca}$ ) was used to detect fluorescence of the rhodamine $6 \mathrm{G}$ with excitation achieved using an internal laser emitting at $\lambda=532 \mathrm{~nm}$.

The air amplifier desolvation study was carried out after replacement of the standard Thermo MS capillary with an extended capillary to allow penetration of the air amplifier nozzle by $26 \mathrm{~mm}$, in the manner previously described by Hawkridge et al. [15]. The capillary temperature was set to $300{ }^{\circ} \mathrm{C}$ and was controlled by the instrument without significant deviation from the set point. The air amplifier had no voltage bias applied throughout this set of experiments. Additionally, two heating coils (part no. 03,122-30; Cole-Parmer Instru- 
ment Company, Vernon Hills, IL) were wrapped around the air amplifier body and a portion of copper tubing (which connected the air amplifier to the polyethylene tubing from the nitrogen regulator). The temperature of the heating coils was controlled by a Powerstat variable autotransformer (Warner Electric, Bristol, CT), and the temperature of the air amplifier body was monitored using a Fluke thermocouple (part no. 80PJ-1; Everette, WA) attached to a Fluke $87 \mathrm{~V}$ multi-meter.

\section{Results and Discussion}

Figure $2 \mathrm{a}$ shows the screen imaged using a BioRad fluorescence scanner following exposure to electrospray ionization of rhodamine $6 \mathrm{G}$. Figure $2 \mathrm{a}$ is a fluorescence image of the screen used to quantify the ESI cross section. The fluorescence intensity legend is shown at the right indicating the range from zero to $>75$ arbitrary units of fluorescence. The screen demonstrates no detectable fluorescence on the portion where the air amplifier was OFF (Figure 2a, left) for 5 min of continuous electrospray. However, when the air amplifier was turned ON (Figure 2a, right) accompanied with 5 min of continuous electrospray, an intense region of fluorescent dye is observed. The spatial droplet/ion

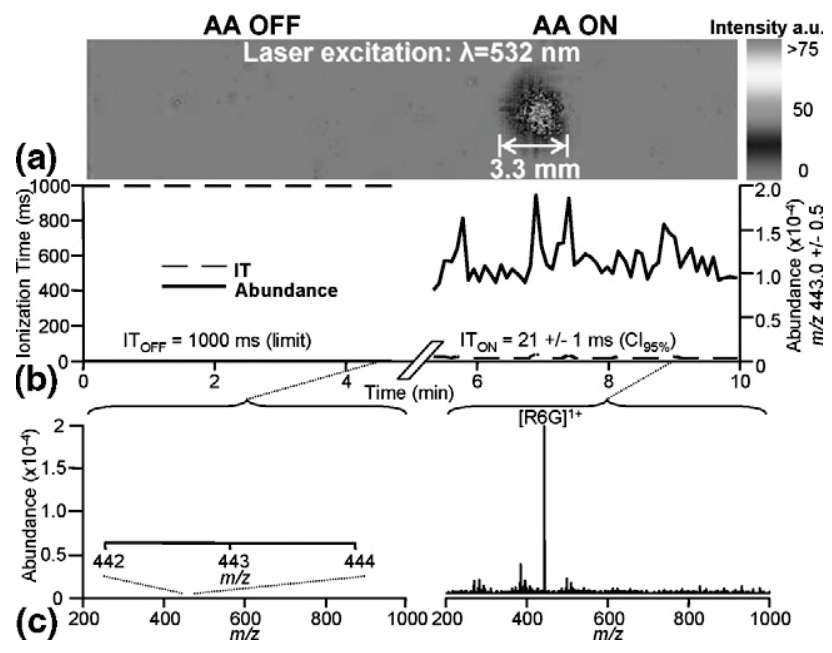

Figure 2. (a) Fluorescence image of the stainless steel mesh screen following electrosprayed rhodamine $6 \mathrm{G}$ containing droplets/ions, as detected by a BioRad imager (intensity key at right of imaged screen). No fluorescence is observed with the air amplifier OFF (left). A tightly spotted region of intense fluorescence is observed with the air amplifier ON (right), providing a measure of the area of focused electrosprayed ions. (b) With the air amplifier OFF (left, screen in place) the ion injection time reaches the maximum AGC time limit of $1000 \mathrm{~ms}$, yet the ion abundance remains below the detectable limit. When the air amplifier is turned $\mathrm{ON}$ (right, screen in place), the ion injection time decreases to $21 \mathrm{~ms}$ and significant ion abundance is observed. (c) The FT-ICR mass spectrum observed with the air amplifier OFF (left, screen in place) demonstrates that no MS signal is observed without aid from the air amplifier, however, a clean spectrum of rhodamine 6G ions is observed with the assistance of an air amplifier (right, screen in place). focusing by the air amplifier is visually confirmed by the fluorescent image of electrosprayed ions.

The area of the detected fluorescence spot was determined using the Quantity One software provided by BioRad, Inc., yielding a tight spot of electrosprayed ions covering an area $\sim 33.5 \mathrm{~mm}^{2}$ with an equivalent diameter of $\sim 3.3 \mathrm{~mm}$. This presents a direct method to quantify the area coverage (i.e., a measure of the device focusing efficiency) of the electrosprayed ions at the outlet of the air amplifier. The maximum cross sectional area potentially available for sample transport with a standard 550 micron i.d. transfer capillary is $0.95 \mathrm{~mm}^{2}$, which would result in $\sim 3 \%$ of the electrosprayed droplets/ions matched to the capillary cross sectional area, given a uniform spatial distribution within the spot. With the air amplifier turned OFF, this ratio, which is a measure of the ion transmission efficiency, is vanishingly small, resulting in no detectable MS signal. Although the configuration of the screen outside of the air amplifier is rudimentary, it clearly demonstrates the nitrogen focusing by the air amplifier is fully responsible for the observed fluorescence spot.

Figure $2 \mathrm{~b}$ shows the ion injection times and selected ion chromatogram $(m / z=443.0 \pm 0.5)$ with respect to time allocated for electrospray. The total time of continuous electrospray was $10 \mathrm{~min}$, during which the air amplifier was left OFF for the first $5 \mathrm{~min}\left(\mathrm{IT}_{\mathrm{OFF}}\right)$, and was turned $\mathrm{ON}$ for the last $5 \mathrm{~min}\left(\mathrm{IT}_{\mathrm{ON}}\right)$. In the data analysis it was necessary to account for a short period of time just after the air amplifier was turned $\mathrm{ON}$ to allow the pressure within the air amplifier to reach steadystate. The selected ion chromatogram was boxcar smoothed $(n=7)$ before analysis. In the first half of the chromatogram (air amplifier OFF), the ion injection time reaches the maximum level and no MS signal is observed (FT mode). A dramatic improvement is realized after the air amplifier is turned ON ensuing reduced ion injection time and an abundant MS signal (FT mode). The improvement in ion injection time $\left(\mathrm{IT}_{\mathrm{ON}} / \mathrm{IT}_{\mathrm{OFF}}\right)$ resulting from use of the air amplifier can only be estimated since $\mathrm{IT}_{\mathrm{OFF}}$ is limited by the preset MS ion injection time limit of $1000 \mathrm{~ms}$ when the air amplifier is OFF. This significant decrease in ion injection time from $\mathrm{IT}_{\mathrm{OFF}}=1000 \mathrm{~ms}$ to $\mathrm{IT}_{\mathrm{ON}}=21 \mathrm{~ms}$ $\left(\mathrm{RSD}_{\mathrm{ON}}=18 \%\right)$ resulting from use of the air amplifier confers the ability to obtain more MS or MS/MS per unit time, leading to improved MS throughput which is particularly advantageous for proteomic applications.

Figure 2c shows no MS signal with the air amplifier OFF (left), further supported by an expanded view of the singly charged rhodamine $6 \mathrm{G}$ region (FT mode). This is due to the limited droplet/ion transport associated with standard nanoelectrospray (without the air amplifier). By turning the air amplifier ON (Figure 2c, right) spectra with low chemical background and high signal-to-noise ratio are observed.

Subsequent experiments were carried out to investigate the signal attenuation by insertion of the screen between the emitter and the capillary. This was accom- 
plished by comparing the ion abundance without the screen in place, to the ion abundance with the screen in place. The screen was positioned $\sim 2 \mathrm{~mm}$ from the MS inlet for each experiment and parallel with the front of the instrument. At an emitter to capillary distance of 25 $\mathrm{mm}$, the observed signal was attenuated by $\sim 95 \%$ with the screen in place. By increasing the distance to $40 \mathrm{~mm}$, it is expected that the attenuation will increase approaching $100 \%$ (without the air amplifier). With the air amplifier $\mathrm{ON}$, the screen attenuation at a $40 \mathrm{~mm}$ emitter to MS inlet distance was experimentally found to be $\sim 20 \%$. The screen effects on the gas flow profile of the air amplifier and focusing will be primarily local (near the mesh screen) and do not affect the overall gas dynamics. This is attributed to the high permeability of the screen and the relatively high flow rate used in the air amplifier resulting in efficient mixing through the device. An additional observation was that the dispersity of the fluorescent dye on the screen increased as a function of emitter distance from the screen. This observation is expected, as Coulombic repulsions and gas-phase interactions have more time to expand the sample plume as it travels from emitter to MS capillary. These experiments also verified that abundant ESI signal can be attained without the air amplifier or screen in place with a normal ESI emitter to MS inlet distance $(\sim 2 \mathrm{~mm})$.

Figure 3 demonstrates the analyte response under various conditions. Electrospray has previously been demonstrated to have a selective response based on solute physicochemical properties [18]. Tetrabutylammonium $\left(^{*}\right)$ and tetraheptylammonium $\left(^{* *}\right)$ are depicted on the left

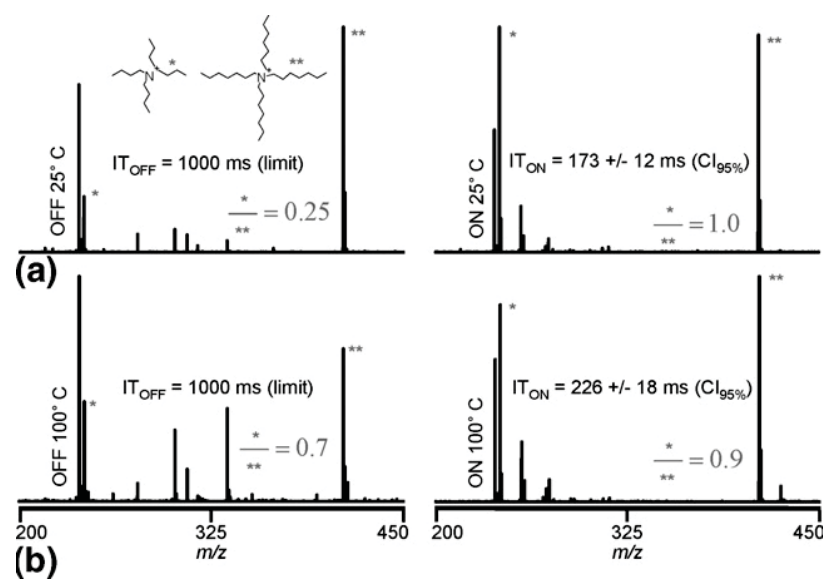

Figure 3. (a) Analysis of tetrabutyl $\left(^{*}\right)$ and tetraheptyl (**) ammonium halide. The response in standard ESI at room temperature results in a much lower response of tetrabutyl compared with tetraheptyl based on the much higher solvophobicity of tetraheptyl ammonium halide. With the air amplifier $\mathrm{ON}$ at room temperature, an equivalent response of the two molecules is observed, resulting in a true indication of the concentration of each molecule within the bulk solution. (b) The biased response observed in ESI is also minimized with the addition of heat as the body of the air amplifier and the inlet nitrogen is heated to $100{ }^{\circ} \mathrm{C}$. With heat and the air amplifier $\mathrm{ON}$, a nearly equivalent response is attained of the tetrabutyl and tetraheptyl ammonium halides. side of Figure 3a. Shown on the left of Figure 3a, the air amplifier is OFF at ambient temperature, and the tetraheptyl has a much stronger response based on its higher solvophobicity as compared with the tetrabutyl ammonium halide. The ion injection time was maximized at $1000 \mathrm{~ms}$ as the AGC limit was not reached without the air amplifier. The air amplifier $\mathrm{ON}$ at ambient temperature (Figure 3a, right) yields a unity analyte response, thus providing a true indication of the specific component concentration within a solution of mixed analyte. The ion injection time of $173 \mathrm{~ms}$ ( RSD $=17 \%$ ) was lowest in this scenario. By heating the body of the air amplifier (Figure 3b, left), but leaving the device OFF, an increase in the tetrabutyl abundance was observed, pointing to increased desolvation by means of convective heat transfer. This observation is in agreement with previous reports of heating the electrospray source to normalize the analyte response [20]. Even with improved desolvation, the AGC limit was not met, and the ion injection time was maximized to $1000 \mathrm{~ms}$. On the right of Figure $3 \mathrm{~b}$ it is shown that with the air amplifier $\mathrm{ON}$ at a temperature of $100{ }^{\circ} \mathrm{C}$ resulted in a nearly equivalent analyte response. The ion injection time with the air amplifier $\mathrm{ON}$ and at $100{ }^{\circ} \mathrm{C}$ was reduced to 226 ms $(\operatorname{RSD}=19 \%)$, indicating increased ion abundance. This is the first demonstration of heating the air amplifier while simultaneously increasing ion abundance and, thus, leaves room for optimizing the implementation of the device while heating. By eliminating electrospray bias due to physicochemical properties, a true prediction of the analyte concentration within a mixture is possible.

The reported experiments support the hypothesis that there is a dual mechanism involving both aerodynamic focusing of electrosprayed droplets/ions for the increased ion abundance achieved by the air amplifier and improved sample desolvation [14, 16]. The air amplifier provides increased ion abundance to the detector, resulting in reduced ion injection times, which allows for shorter analysis times and duty cycles. Improvements to the current air amplifier design, resulting in a tighter ion beam (i.e., smaller cross-sectional area of electrosprayed ions), can be appreciated in mass spectrometry, yielding higher ion transmission efficiency, better signal-to-noise ratio for MS detection, and faster data acquisition. The current empirically optimized design allows for reduced ion injection time and improves the capture efficiency compared with standard electrospray. This work demonstrates an effective method to experimentally evaluate new air amplifier designs by combination of mass spectrometry (via measurement of the ion injection time ratio) and an orthogonal method, fluorescence spectroscopy (via measurement of an area occupied by the focused electrospray ions). Continued efforts to model and simulate droplet and ion transport in the air amplifier will enable improved designs and device implementation to further enhance ion transmission efficiency and lower limits of detection. The improvements shown in this work for 
standard ESI-MS should be also of great value to other emerging ambient ionization techniques such as DESI $[21,22]$, MALDESI [23, 24], and AMUSE [25, 26].

\section{Acknowledgments}

The authors gratefully acknowledge the financial support received from the National Institutes of Health (R33 CA105295, R21 RR021474-01A1), the W. M. Keck Foundation, and North Carolina State University.

\section{References}

1. Fenn, J. B.; Mann, M.; Meng, C. K.; Wong, S. F.; Whitehouse, C. M. Electrospray Ionization for Mass-Spectrometry of Large Biomolecules. Science 1989, 246(4926), 64-71.

2. Smith, R. D.; Loo, J. A.; Edmonds, C. G.; Barinaga, C. J.; Udseth, H. R. New Developments in Biochemical Mass-Spectrometry-Electrospray lonization. Anal. Chem. 1990, 62(9), 882-899.

3. Kebarle, P.; Tang, L. From Ions in Solution to Ions in the Gas-Phase- the Mechanism of Electrospray Mass-Spectrometry. Anal. Chem. 1993, 65(22), A972-A986.

4. Tang, L.; Kebarle, P. Dependence of Ion Intensity in Electrospray Mass-Spectrometry on the Concentration of the Analytes in the Electrosprayed Solution. Anal. Chem. 1993, 65(24), 3654-3668.

5. Zook, D. R. Bruins, A. P. On Cluster Ions, Ion Transmission, and Linear Dynamic Range Limitations in Electrospray (Ionspray) Mass Spectrometry. Int. J. Mass Spectrom. Ion Phys. 1997, 162(1/3), 129-147.

6. Cech, N. B.; Enke, C. G. Practical Implications of Some Recent Studies in Electrospray Ionization Fundamentals. Mass Spectrom. Rev. 2001, 20(6), 362-387.

7. Page, J. S.; Kelly, R. T.; Tang, K.; Smith, R. D. Ionization and Transmission Efficiency in an Electrospray Ionization-Mass Spectrometry Interface. J. Am. Soc. Mass Spectrom. 2007, in press.

8. Shaffer, S. A.; Tang, K. Q.; Anderson, G. A.; Prior, D. C.; Udseth, H. R.; Smith, R. D. A Novel Ion Funnel for Focusing Ions at Elevated Pressure Using Electrospray Ionization Mass Spectrometry. Rapid Commun. Mass Spectrom. 1997, 11(16), 1813-1817.

9. Him, T.; Tolmachev, A. V.; Harkewicz, R.; Prior, D. C.; Anderson, G.; Udseth, H. R.; Smith, R. D.; Bailey, T. H.; Rakov, S.; Futrell, J. H. Design and Implementation of a New Electrodynamic Ion Funnel. Anal. Chem. 2000, 72(10), 2247-2255.

10. Kim, T.; Udseth, H. R.; Smith, R. D. Improved Ion Transmission from Atmospheric Pressure to High Vacuum Using a Multicapillary Inlet and Electrodynamic Ion Funnel Interface. Anal. Chem. 2000, 72(20), 50145019.

11. Buryakov, I. A.; Krylov, E. V.; Nazarov, E. G.; Rasulev, U. K. A New Method of Separation of Multi-Atomic Ions by Mobility at Atmospher-
ic-Pressure Using a High-Frequency Amplitude-Asymmetric Strong Electric-Field. Int. J. Mass Spectrom. Ion Phys. 1993, 128(3), 143-148.

12. Purves, R. W.; Guevremont, R. Electrospray Ionization High-Field Asymmetric Waveform Ion Mobility Spectrometry-Mass Spectrometry. Anal. Chem. 1999, 71(13), 2346-2357.

13. Barnett, D. A.; Ells, B.; Guevremont, R.; Purves, R. W. Application of ESI-FAIMS-MS to the Analysis of Tryptic Peptides. J. Am. Soc. Mass Spectrom. 2002, 13(11), 1282-1291.

14. Zhou, L.; Yue, B. F.; Dearden, D. V.; Lee, E. D.; Rockwood, A. L.; Lee, M. L. Incorporation of a Venturi Device in Electrospray Ionization. Anal. Chem. 2003, 75(21), 5978-5983.

15. Hawkridge, A. M.; Zhou, L.; Lee, M. L.; Muddiman, D. C. Analytical Performance of a Venturi Device Integrated into an Electrospray Ionization Fourier Transform Ion Cyclotron Resonance Mass Spectrometer for Analysis of Nucleic Acids. Anal. Chem. 2004, 76(14), 4118-4122.

16. Yang, P. X.; Cooks, R. G.; Ouyang, Z.; Hawkridge, A. M.; Muddiman, D. C. Gentle Protein Ionization Assisted by High-Velocity Gas Flow. Anal. Chem. 2005, 77(19), 6174-6183.

17. Zhou, L.; Zhai, L. L.; Yue, B. F.; Lee, E. D.; Lee, M. L. New Interface Plate for Microspray Ionization Mass Spectrometry. Anal. Bioanal. Chem. 2006, 385(6), 1087-1091.

18. Fenn, J. B. Ion Formation from Charged Droplets-Roles of Geometry, Energy, and Time. J. Am. Soc. Mass Spectrom. 1993, 4(7), 524-535.

19. Marshall, A. G.; Hendrickson, C. L. Charge Reduction Lowers Mass Resolving Power for Isotopically Resolved Electrospray Ionization Fourier Transform Ion Cyclotron Resonance Mass Spectra. Rapid Commun. Mass Spectrom. 2001, 15(3), 232-235.

20. Frahm, J. L.; Muddiman, D. C. Leveling Response Factors in the Electrospray Ionization Process Using a Heated Capillary Interface. J. Am. Soc. Mass Spectrom. 2005, 16(5), 772-778.

21. Takats, Z.; Wiseman, J. M.; Gologan, B.; Cooks, R. G. Mass Spectrometry Sampling Under Ambient Conditions with Desorption Electrospray Ionization. Science 2004, 306(5695), 471-473.

22. Bereman, M. S.; Nyadong, L.; Fernandez, F. M.; Muddiman, D. C. Direct High-Resolution Peptide and Protein Analysis by Desorption Electrospray Ionization Fourier Transform Ion Cyclotron Resonance Mass Spectrometry. Rapid Commun. Mass Spectrom. 2006, 20(22), 3409-3411.

23. Sampson, J. S.; Hawkridge, A. M.; Muddiman, D. C. Generation and Detection of Multiply-Charged Peptides and Proteins by MatrixAssisted Laser Desorption Electrospray Ionization (MALDESI) Fourier Transform Ion Cyclotron Resonance Mass Spectrometry. J. Am. Soc. Mass Spectrom. 2006, 17(12), 1712-1716.

24. Sampson, J. S.; Hawkridge, A. M.; Muddiman, D. C. Direct Characterization of Intact Polypeptides by Matrix-Assisted Laser Desorption Electrospray Ionization Quadrupole Fourier Transform Ion Cyclotron Resonance Mass Spectrometry. Rapid Commun. Mass Spectrom. 2007, 21(7), 1150-1154.

25. Meacham, J. M.; Ejimofor, C.; Kumar, S.; Degertekin, F. L.; Fedorov, A. G. Micromachined Ultrasonic Droplet Generator Based on a Liquid Horn Structure. Review of Scientific Instruments 2004, 75(5), 1347-1352.

26. Aderogba, S.; Meacham, J. M.; Degertekin, F. L.; Fedorov, A. G. Fernandez, F. M. Nanoelectrospray Ion Generation for High-Throughput Mass Spectrometry Using a Micromachined Ultrasonic Ejector Array. Applied Phys. Lett. 2005, 86(20), 203110-203113. 